\title{
APPLICATION OF RELIABLE CHANGE INDICES TO COMPUTERIZED NEUROPSYCHOLOGICAL MEASURES OF CONCUSSION
}

\section{THOMAS D. PARSONS}

Institute for Creative Technologies

University of Southern California

California, USA

and

School of Gerontology

University of Southern California

California, USA

\author{
ANDREW J. NOTEBAERT \\ University of lowa \\ lowa, USA \\ EDGAR W. SHIELDS \\ KEVIN M. GUSKIEWICZ \\ University of North Carolina at Chapel Hill \\ North Carolina, USA
}

\footnotetext{
Serial assessments of neurocognitive functioning in athletes with concussion are commonly used for return to play decisions. This study provides reliable change indices (RCIs) for computerized tests from 40 NCAA Division I collegiate athletes that suffered a sports-related concussion. The normative data that resulted from the RCIs and subsequent analyses of differences between improved and not improved
}

Received 8 November 2007.

Address correspondence to Thomas D. Parsons, Ph.D., Research Scientist, Neuropsychologist, Co-Director VRPSYCH Lab, Assistant Research Professor of Gerontology, University of Southern California, Institute for Creative Technologies, 13274 Fiji Way, Marina del Rey, CA 90292-4019, USA. E-mail: tparsons@usc.edu 
athletes may aid both clinicians and researchers to assess whether observed change on neuropsychological measures is reliable change or change due simply to practice effects. Hence, the RCIs presented herein provide information that may be used judiciously by a clinician for assessing meaningful change.

Keywords neurocognitive, reliable change, sports-related concussion, statistical assessment

\section{INTRODUCTION}

Assessment of intraindividual change is important for determining the significance of changes in test scores across serial neuropsychological assessments of athletes sustaining a concussion. Persons with sports-related concussion tend to show signs of at least some acute neurocognitive difficulties associated with concussion. However, the nature and course of postacute neurocognitive recovery is variable. Although many sports-related concussions produce a number of subjective symptoms (e.g., headaches, dizziness, wooziness, decreased balance, and coordination, as well as memory impairment), results from a number of studies suggest that for the athletic population most neurocognitive deficits associated with sports-related concussion resolve in the first several days (Bleiberg et al., 2004b; Bruce \& Echemendia, 2003; Macciocchi, Barth, Alves, Rimel, \& Jane, 1996; McCrea, Guskiewicz, \& Marshall, 2003). However, there may be a cumulative effect in which the likelihood of future concussion is increased in athletes who have previously sustained a concussion (Guskiewicz et al., 2003b). Further, repeated exposure to sports-related activities may cause more subtle concussion symptoms (e.g., dizziness, headaches, etc.) (Webbe \& Ochs, 2003; Witol \& Webbe, 2003) and players may be at an elevated risk for repeat concussion during the symptomatic postconcussive period (McCrea et al., 2003). As a result, cerebral concussion in individuals with a history of previous concussion increases the likelihood of more enduring cognitive effects (Moser \& Schatz, 2002).

Since the neurocognitive sequelae of sports-related concussion often present as relatively mild symptoms, baseline computerized testing may have import for the sports-concussion arena. Computerized assessment of athletes' neurocognitive performance has been shown to be a powerful assessment tool for comparing pre- and postconcussion neuropsychological data. This repeated measures assessment allows the neuropsychologist to establish changes in neurocognitive status as a result of the concussion and evaluate the degree of symptom resolution. Additionally, computerized neurcognitive assessments have value over standard paper and pencil in that they are less time-consuming 
to allow for a wide-based, baseline-testing program (Kane \& Reeves, 1997; Mead \& Drasgow, 1993). As a result, persons interested in a baseline-testing paradigm may prefer computerized measures for identifying neurocognitive deficits, tracking recovery progress, and assisting in return-to-play decisions (Schatz \& Zillmer, 2003).

The use of standardized computer neurocognitive tests for athletes sustaining a concussion has been recommended by several sports medicine bodies (Aubry, Cantu, \& Dvorak, 2001; Guskiewicz, Bruce, \& Cantu, 2004; McCrory, Johnston, \& Meeuwisse, 2005) but the usefulness and application of such tests is still debated among clinicians and researchers (Ferrara, McCrea, Peterson, \& Guskiewicz, 2001; Zillmer, 2003).

The National Athletic Trainers' Association's position statement on management of sports-related concussion recently recommended the use of standardized measures of concussion assessment (SMCA) to aid clinicians in making better informed decisions regarding concussion assessment and return to play considerations (Guskiewicz et al., 2004). Despite these recommendations, clinicians have not widely adopted these standardized measures in practice (Ferrara et al., 2001; Notebaert \& Guskiewicz, 2005).

Studies relying on pre-post comparisons tend to demonstrate smaller effects, likely due to practice effects with repeated administration. Whilst the comparison of an athlete's performance following a concussion to the athlete's baseline performance may be helpful to decrease variance, effect-size estimates drawn from repeated administrations of neuropsychological exams likely represent an underestimate of sports-concussion effects due to practice effects (Belanger \& Vanderploeg, 2005; Wilson, Watson, Baddeley, Emslie, \& Evans, 2000).

Research studying SMCA and their effectiveness has primarily involved analyses of group means (differences between injured and noninjured athletes). Many of these studies have shown the decline and recovery of scores on neuropsychological tests, postural stability tests, and symptom checklist scores following concussion (Bleiberg, Cernich, \& Cameron, 2004a; Guskiewicz, McCrea, \& Marshall, 2003a; Iverson, Gaetz, Lovell, \& Collins, 2004; McCrea et al., 2003; Moser, Schatz, \& Jordan, 2005; Valovich-McLeod et al., 2004). Statistical analysis usually involves $t$-tests or analysis of variance to determine group differences. While this approach is helpful as a research tool in describing trends in concussion recovery, it is limited in its ability to help clinicians, such as sports medicine physicians and athletic trainers, evaluate and understand individual differences often associated with cerebral concussion. 
The matter of whether a given athlete has deteriorated cognitively following concussion may be more relevant than the issue of whether a group of athletes with concussion has shown cognitive change. In situations where clinicians have access to only two measurements, a "simple change score" may be calculated, in which the results of criterion variables at time one (T1) and time two (T2) are assessed (i.e., baseline and follow up). For example, a clinician may use the simple change score method to calculate an athlete's performance on a psychomotor task. Unfortunately, the simple change method proffers no definitive criterion above or below which determination of "significant change" may be made. Precise decision making based on information gleaned from simple change scores is further hampered by the fact that this method is severely limited by lack of statistical adjustment for practice effects.

Use of reliable change indices (RCIs) provide a criterion by which the clinician may establish that an observed change is a meaningful change. Specifically, an RCI greater than +1.645 is likely to occur randomly in only $5 \%$ of cases $(p<.05)$, and is thus considered a significant change, while reliable decline occurs when values fall below -1.645 . Further, RCIs help adjust for the reliability of a given test. As a result, a test with less reliability requires a larger test-retest difference score to be interpreted as significant. The classical RCI method included a numerator with the ratio of this estimation and a denominator with standard error used as a criterion (Jacobson \& Truax, 1991).

An adjusted RCI may be calculated to control for practice effects in which the predicted score at postinjury is the athlete's baseline score plus the mean practice effect for the normative sample (Chelune, Naugle, Luders, \& Awad, 1991; Frerichs \& Tuokko, 2005; Heaton, Temkin, \& Dikmen, 2001). The adjusted RCI has been found to perform comparably to more complex regression formulas (Frerichs \& Tuokko, 2005; Heaton et al., 2001; Temkin, Heaton, Grant, \& Dikmen, 1999). This adjusted RCI may be used to aid the clinician in assuring that an observed change in a concussion sufferer does not reflect chance or measurement error. In situations where an RCI exceeds (in either a positive or negative direction) a decided upon segment of the normal distribution, the observed change is judged to be a reliable improvement or deterioration on a given criterion.

Application of RCIs to SMCAs may be beneficial in advancing SMCA use in clinical applications. Our purpose for this study is to demonstrate RCI calculation and interpretation of results from three concussion assessment measures, including neuropsychological tests, postural stability tests, and symptom checklist scores. 


\section{METHODS}

\section{Subjects}

Forty NCAA Division 1 collegiate athletes (24 male, 16 female) sustaining a sports-related concussion participated in this study. Athletes participated in a variety of sports including football $(n=14)$, men's soccer $(n=4)$, men's lacrosse $(n=2)$, wrestling $(n=4)$, women's soccer $(n=9)$, women's lacrosse $(n=3)$, women's basketball $(n=3)$, and track and field ( $n=1$, pole vaulter). All participants were assessed using a computerized neuropsychological test battery, computerized force plate posturography, and a symptom checklist prior to and after a concussion; however, one athlete did not complete postural stability testing postconcussion. All of the testing sessions were completed between 2001 and 2005. All athletes read and signed an informed consent document approved by the University's Institutional Review Board prior to being baseline tested. Demographics describing the athletes and the time between baseline and injury are presented in Table 1 .

\section{Test Measures}

All participants were tested at baseline and postconcussion on three standardized tests currently used by the University's sports medicine staff according to an established concussion evaluation protocol. Performance on these tests aids the sports medicine staff in determining whether an athlete can return to play with limited participation in his or her sport. These tests include the Automated Neuropsychological Assessment Metrics (ANAM), the Sensory Organization Test (SOT), and a Graded Symptom Checklist (GSC). Tests were given by one of the several certified athletic trainers who were experienced with administering these tests and worked in the Sports Medicine Research Laboratory at the university. Standardized instructions were given to all of the athletes to minimize the effects of multiple administrators. There was no fixed order for test administration.

Automated Neuropsychological Assessment Metrics. The ANAM (National Rehabilitation Hospital Assistive Technology and Neuroscience Center, Washington, DC) is a computerized neuropsychological test battery with six modules for various cognitive domains (one module is repeated). The battery takes approximately $20 \mathrm{~min}$ to complete on a desktop personal computer. The test battery included the following modules: simple reaction time (SRT), math processing (MATH), Sternberg memory (SM6), matching to sample (MTS), 


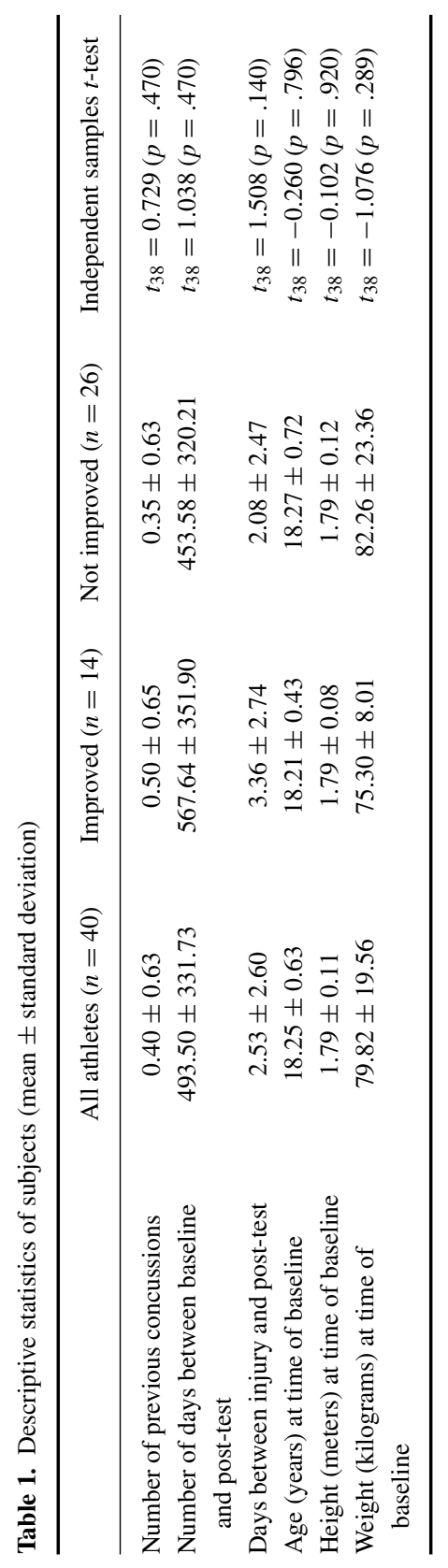


procedural reaction time (PRT), and code substitution (CDS). The SRT module was taken twice, once at the beginning of the test and once at the end (SRT1 and SRT2). These modules assess reaction time (SRT and PRT), working memory (SM6), visual memory (MTS), mental processing speed and efficiency (MATH), and attention (CDS), all of which are commonly affected by concussion (Bleiberg, Halpern, Reeves, \& Daniel, 1998; Grindel, Lovell, \& Collins, 2001; McCrea, Kelly, Randolph, Cisler, \& Berger, 2002).

Each athlete completed ANAM on a computer and all athletes received standardized instructions prior to beginning the testing. The athlete reacted to stimuli presented on the computer screen by clicking the left or right mouse button. The computer assessed speed and accuracy of the athletes by calculating a throughput score for each module. A higher throughput score indicated a better performance. The test automatically ran each module in the same order but the time between stimulus and type of stimuli differed with the postconcussion administration to minimize practice effects.

Sensory Organization Test. The SOT is a computerized measure of postural stability that was conducted using the NeuroCom Smart Balance Master (NeuroCom International, Clackamas, OR, USA). The athlete was fit into a harness and then placed in the NeuroCom onto a dual forceplate system. The athlete was given instructions as to the workings of the test prior to the test. The test protocol consisted of 18 trials lasting $20 \mathrm{~s}$ each. The athletes were instructed to remain as still as possible during the trial and to maintain their balance. There were six different test conditions with three trials each. Three different visual conditions (eyes open, eyes closed, or sway visual reference) were crossed with two different surface conditions (fixed or sway surface). A complete description of this postural stability testing has been previously reported in the literature (Cavanaugh et al., 2005; Guskiewicz, Ross, \& Marshall, 2001).

The SOT computer program calculated a percentage score for each trial and then a composite score (SOTcomp) for overall postural stability. Higher composite scores indicated better ability to maintain a stable and still position during the trials. The computer also calculated three ratios based on the ability to use vestibular (SOTvest), somatosensory (SOTsom), and visual (SOTvis) pathways to maintain balance (Guskiewicz, 2001).

Graded Symptom Checklist. Symptom information was acquired using a modified version of the GSC (Lovell \& Collins, 1998), an 18-item symptom list graded on a 7-point Likert scale. These symptoms, which are normally associated with concussion, included but were not limited to headache, 
nausea, dizziness, trouble sleeping, difficulty concentrating, and difficulty remembering. The athlete graded each symptom on a $0-6$ scale with 0 indicating not present, 1 indicating mild, 3 indicating moderate, and 6 indicating severe. During baseline measurement, the athlete was asked to recall if he or she regularly experienced the symptom, approximately three or more times a week. If he or she did, they were asked to rate the average symptom severity over that time period. A total symptom score was calculated by summing the score for each of the symptoms. Higher scores indicated an increase in global symptom severity.

After an athlete sustained a concussion, they were reassessed on the GSC to indicate current symptom severity. The athlete indicated the current severity for each of the same 18 items on the same Likert scale. A total symptom score was calculated by summing all of the scores given for his or her current condition.

\section{Data Analytic Considerations}

Data were analyzed using SPSS for Windows (version 13.0; SPSS Inc., Chicago, IL, USA). All alpha levels were set to .05 a priori. Change scores, correcting for measurement error and practice effects, were calculated for each participant using a previously developed RCI methodology (Heaton, et al., 2001; Woods et al., 2006). All test difference scores were normally distributed. To ensure that all positive z-scores indicated improvement and negative scores denoted a decline in performance, we multiplied the total symptom score by -1 .

The RCI method used to correct for measurement error and practice effects was defined as $\left(\left(X_{2}-X_{1}\right)-\left(M_{2}-M_{1}\right)\right) / \mathrm{SDD}$, where $X_{1}$ was the observed pretest score, $X_{2}$ was the observed post-test score, SDD was the standard deviation of the group test-retest difference, $M_{1}$ was the group mean pretest score, and $M_{2}$ was the group mean post-test score. Practice effect correction involves the addition of a constant that is based upon the group-level average change (Heaton et al., 2001; Woods et al., 2006). For the purposes of these analyses, practice effects were apparent in those situations in which group mean post-test score greater than group mean pretest score represents mean gain, and group mean post-test score less than group mean pretest score represents mean decline. Reliable improvement occurred when values exceeded 1.645 and reliable decline when values fell below -1.645 .

RCIs were developed for two groups. The first group (improved) comprised athletes that took the SMCA following a concussion. Based on 
their performance on the SMCA tests, we classified them as improved based on certain criteria. The criterion for classification was acknowledged as achieving $95 \%$ of baseline or better on each of the three standardized tests. The $95 \%$ of baseline criteria was one that was used for clinical decision making at the time of test administration. For the ANAM test, the athlete was improved if he or she achieved $95 \%$ of baseline or higher for six of the seven parts. It is important to note that classification into the improved group was done by the researchers and not by the sports medicine team, although we tried to mimic as closely as we could to return to play decisions that were made using SMCA results as part of the decision process. Clinical decisions were made by the team physician in consultation with members of the sports medicine staff who had experience in using these standardized tests, as well as experience in working with the athlete being treated.

The second group (not improved) was comprised of athletes who were concussed, received SMCA, and were to return for repeat follow-up testing the next day. These athletes did not initially score above $95 \%$ of their baseline scores for each of the SMCA after the first testing session done postconcussion and were to refrain from participating in practice or competition in their respective sport. Athletes continued to be tested every day until baseline levels were reached. Again, classification for this study was done by the researchers based on the athletes returning for additional follow-up testing. Even though follow-up testing was done, only data from the athletes' initial testing was used in this analysis.

\section{RESULTS}

The distribution of scores in the improved group revealed cut scores for each cognitive measure that provide a $90 \%$ confidence interval for classifying change. Mean practice effects, SDD, and confidence intervals for the improved group are shown in Table 2. For the improved group, two ANAM modules (SRT1 and MTS) showed a decrease in performance. Symptom scores were increased but less than the entire group. The SOT scores improved for composite score compared to baseline, but the somatosensory ratio still indicated a slight decrease.

The distribution of scores in the not improved group revealed cut scores for each cognitive measure that provide a $90 \%$ confidence interval for classifying change. Mean practice effects, SDD, and confidence intervals for the not improved group are shown in Table 3. There were decreases in performance for all SMCA excluding the Math, PRT, and CDS modules of ANAM. Average 
Table 2. Confidence intervals and percentage of change for "improved" subjects

\begin{tabular}{lcrrrrrr}
\hline Test & \%Imp & \%Dec & $\%$ NC & CI + & CI - & Mean & SDD \\
\hline ANAM $(n=14)$ & & & & & & & \\
SRT1 & 0 & 7 & 93 & 41.21 & -48.38 & -3.59 & 27.31 \\
SRT2 & 0 & 14 & 86 & 57.74 & -51.01 & 3.37 & 33.16 \\
MATH & 0 & 7 & 93 & 9.60 & -4.30 & 2.65 & 4.24 \\
SM6 & 7 & 7 & 86 & 28.54 & -18.13 & 5.21 & 14.23 \\
MTS & 0 & 7 & 93 & 15.09 & -18.36 & -1.64 & 10.20 \\
PRT & 7 & 0 & 93 & 35.04 & -8.50 & 13.27 & 13.27 \\
CDS & 7 & 0 & 93 & 14.92 & -12.80 & 1.06 & 8.45 \\
Symptom total $(n=14)$ & 0 & 7 & 93 & -17.81 & 13.24 & -2.29 & 9.47 \\
SOT $(n=14)$ & & & & & & & \\
SOTcomp & 0 & 7 & 93 & 6.59 & -4.41 & 1.09 & 3.35 \\
SOTvest & 0 & 7 & 93 & 0.16 & -0.13 & 0.01 & 0.09 \\
SOTsom & 0 & 7 & 93 & 0.08 & -0.11 & -0.02 & 0.06 \\
SOTvis & 0 & 14 & 86 & 0.10 & -0.08 & 0.01 & 0.06 \\
\hline
\end{tabular}

Note: $\%$ Imp $=$ the percentage of scores that were statistically improved from baseline, $\% \mathrm{Dec}=$ the percentage of scores that were statistically declined from baseline, $\% \mathrm{NC}=$ the percentage of scores that showed no statistical change from baseline, SDD $=$ standard deviation of the difference, which measures the average error between repeated testing.

symptom score reported was higher than both the whole group and the improved group.

\section{DISCUSSION}

The normative data that resulted from the RCIs and subsequent analyses of differences between improved and not improved athletes may aid both clinicians and researchers to assess whether observed change on neuropsychological measures is reliable change or change due simply to practice effects. These data were derived for SMCAs that are frequently used in assessing athletes. The resulting RCIs provide information that may be used judiciously by a clinician for assessing meaningful change.

The purpose of this study was to demonstrate RCI calculations on commonly used SMCAs. Presented RCI calculations are for a concussed athletic population that were labeled as improved and not improved on the basis of how the RCI confidence intervals classified SMCA results (Tables 2 and 3). Clinicians may judiciously use these confidence intervals to help classify future athletes that have suffered a concussion as improved or declined based on these same SCMA. 
Table 3. Confidence intervals and percentage of change for "not improved" subjects

\begin{tabular}{lrrrrrrr}
\hline Test & \% Imp & $\%$ Dec & $\%$ NC & CI + & CI - & Mean & SDD \\
\hline ANAM $(n=26)$ & & & & & & & \\
SRT1 & 4 & 8 & 88 & 78.04 & -85.24 & -3.60 & 49.78 \\
SRT2 & 0 & 4 & 96 & 80.27 & -83.16 & -1.45 & 49.83 \\
MATH & 8 & 4 & 88 & 9.53 & -7.23 & 1.15 & 5.11 \\
SM6 & 0 & 4 & 96 & 31.79 & -37.30 & -2.76 & 21.06 \\
MTS & 4 & 0 & 96 & 32.55 & -34.80 & -1.13 & 20.53 \\
PRT & 4 & 0 & 96 & 73.27 & -39.58 & 16.85 & 34.41 \\
CDS & 4 & 4 & 92 & 21.94 & -20.63 & 0.66 & 12.98 \\
Symptom total $(n=26)$ & 8 & 4 & 88 & -34.69 & 14.30 & -10.19 & 14.94 \\
SOT $(n=25)$ & & & & & & & \\
SOTcomp & 0 & 8 & 92 & 18.29 & -32.70 & -7.21 & 15.54 \\
SOTvest & 0 & 8 & 92 & 0.34 & -0.58 & -0.12 & 0.28 \\
SOTsom & 4 & 4 & 92 & 0.07 & -0.12 & -0.03 & 0.06 \\
SOTvis & 4 & 8 & 88 & 0.19 & -0.38 & -0.09 & 0.17 \\
\hline
\end{tabular}

Note: $\%$ Imp $=$ the percentage of scores that were statistically improved from baseline, $\% \mathrm{Dec}=$ the percentage of scores that were statistically declined from baseline, $\% \mathrm{NC}=$ the percentage of scores that showed no statistical change from baseline, SDD $=$ standard deviation of the difference, which measures the average error between repeated testing.

The confidence intervals that were created with the presented RCI calculations indicate that some clinical interpretation of results may not adequately reflect what is actually happening with the athlete. Herein, a 95\% of baseline was used as an indicator of return to baseline performance, as is currently used by some sports medicine staffs. However, the calculated confidence intervals indicate that $95 \%$ of baseline may be too conservative. For example, the reportedly improved group had a range of change scores of over 80 points for the SRT1 module. This range indicates that normal change seen for this module would be much greater than a $5 \%$ decline that would exclude an athlete from participation if the standard $95 \%$ of baseline were used. While using a $95 \%$ of baseline criteria would be very conservative, average practice effects could easily place an athlete within that range and misidentify an athlete suffering deleterious effects from a concussion. This may make a strong statement toward the lack of sensitivity of these measures.

Use of an RCI can be beneficial in other circumstances. Presenting normative postconcussion results and subsequent change scores (from the athlete's baseline performance) with corresponding confidence intervals to the athlete, parent, or coach will aid in categorizing the athlete's performance in relation to normal and abnormal change in performance after sustaining a head 
injury. This would provide quantitative information to help illustrate why an athlete is being returned to or withheld from participation. Further, this may aid decision making in an extreme circumstance where an athlete performs significantly worse on one of these standardized measures. This may indicate that the athlete has greatly decreased in functioning and he or she should be referred for advanced medical evaluation and treatment before being allowed to return to competition.

Clinicians may use the RCI calculations presented here in their concussion protocol. When working with a similar population they could use the confidence intervals reported herein to help them interpret change in athletes following a concussion. Clinicians using alternative SMCA can easily calculate RCI for their own interpretation of results. Researchers and clinicians should develop confidence intervals based on RCI calculations for a variety of tools and for specific populations.

It is important to state that RCI calculations on SMCA should not be used as the exclusive tool for deciding if an athlete ought to return to play after sustaining a concussion. A thorough clinical exam by the medical staff is the most important aspect of concussion assessment and evaluation (Guskiewicz et al., 2004). Use of RCIs of SMCAs can help clinicians make better-informed decisions by giving them information that is not regularly available in clinical examinations. Clinicians should utilize as many tools as possible and as resources allow when examining athletes that have sustained a concussion. Better understanding of SMCA use and interpretation is critical to achieving this goal.

RCIs have previously been calculated in several research studies involving cognitive and symptom data associated with concussed subjects (Barr \& McCrea, 2001; Collie et al., 2004; Erlanger et al., 2001; Hinton-Bayre, Geffen, Geffen, McFarland, \& Friis, 1999). Direct comparison to these studies is not possible due to differences in the types of tests used. We chose to use a simpler RCI calculation than the previous studies since their calculations involve advanced RCI and regression methods that many clinicians without advanced mathematical and statistical backgrounds may find difficult to calculate and interpret. We feel that it is important to present an alternative that can be calculated by incorporating simple math and computer skills and to demonstrate how these methods can easily be interpreted. The confidence intervals for these methods have been previously shown to be comparable (Frerichs \& Tuokko, 2005; Heaton et al., 2001; Temkin et al., 1999) and therefore, the simpler calculations presented here should benefit the average clinician. 
It is important to note that although not statistically significant, there was a mean difference of 1 day between concussion injury and initial follow-up testing between the improved and not improved groups (3.4 days and 2.1 days, respectively). Athletes sustaining a concussion were tested using SMCA as soon as possible following the injury. Differences may have been due to athletes traveling or not reporting the injury to the sports medicine staff promptly.

A possible limitation to the interpretive efficacy of this study is that, due to the retrospective nature of our study, we did not have an available control group to compare our findings. A control group comprised of healthy, nonconcussed collegiate athletes would hopefully strengthen our presented findings. Finding comparable subjects that have similar test-retest intervals would be invaluable to the applications that we have presented here and may help more accurately define any potential practice effects of these measures.

It is also important to note that classification of subjects as improved or not improved was done by the researchers and not the sports medicine staff in charge of the athletes' care. Decision for an athlete to be allowed to return to play was made by the sports medicine staff including the team physician and the staff athletic trainer for that sport. Results of SMCA gathered by the research staff were reported to and interpreted with the sports medicine staff, but the team's medical staff ultimately made the decision for the athlete to be allowed to return to play or to return to for more follow-up testing.

Readers may note that in the use of confidence intervals, athletes may present with an increase in symptoms but still be within the confidence interval. It is generally agreed that athletes with increased symptoms should automatically be excluded from participation. While we agree with this assessment, it is important to state that these athletes were classified based on criteria set by the researchers and do not necessarily reflect decisions made by the sports medicine team in regards to the athletes' participation. Finally, our sample size presents another limitation, especially with the differences in our improved and not improved group with respect to being improved or declined.

In summary, SMCA are important tools that should be available to any clinician working with athletes. By implementing the RCI calculations presented herein for data interpretation or by calculating their own RCIs, clinicians have another tool to aid in the interpretation of individual change. This may help clinicians to effectively use SMCA as part of their toolkit when assessing and evaluating an athlete with a concussion and confidently determining if the athlete can return to play. 


\section{REFERENCES}

Aubry, M., Cantu, R., \& Dvorak, J. (2001). Summary and agreement statement of the First International Conference on Concussion in Sport, Vienna 2001. Recommendations for the improvement of safety and health of athletes who may suffer concussive injuries. British Journal of Sports Medicine, 36, 6-10.

Barr, W. B., \& McCrea, M. (2001). Sensitivity and specificity of standardized neurocognitive testing immediately following sports concussion. Journal of the International Neuropsychological Society, 7, 693-702.

Belanger, H. G., \& Vanderploeg, R. D. (2005). The neuropsychological impact of sports-related concussion: A meta-analysis. Journal of the International Neuropsychological Society, 11, 345-357.

Bleiberg, J., Cernich, A. N., \& Cameron, K. (2004a). Duration of cognitive impairment after sports concussion. Neurosurgery, 54, 1073-1078.

Bleiberg, J., Cernich, A. N., Cameron, K., Sun, W., Peck, K., Ecklund, L. P., et al. (2004b). Duration of cognitive impairment after sports concussion. Neurosurgery, 54, 1073-1080.

Bleiberg, J., Halpern, E. L., Reeves, D., \& Daniel, J. C. (1998). Future directions for the neuropsychological assessment of sports concussion. Journal of Head Trauma Rehabilitation, 13, 36-44.

Bruce, J. M., \& Echemendia, R. J. (2003). Delayed-onset deficits in verbal encoding strategies among patients with mild traumatic brain injury. Neuropsychology, 17, 622-629.

Cavanaugh, J. T., Guskiewicz, K. M., Giuliani, C., Marshall, S., Mercer, V., \& Stergiou, N. (2005). Detecting altered postural control after cerebral concussion in athletes with normal postural stability. British Journal of Sports Medicine, 39, 805-811.

Chelune, G. J., Naugle, R. I., Luders, H., \& Awad, I. A. (1991). Prediction of cognitive change as a function of preoperative ability status among temporal lobectomy patients seen at 6-month follow-up. Neurology, 41, 399-404.

Collie, A., Maruff, P., Makdissi, M., McStephen, M., Darby, D. G., \& McCrory, P. (2004). Statistical procedures for determining the extent of cognitive change following concussion. British Journal of Sports Medicine, 38, 273-278.

Erlanger, D., Saliba, E., Barth, J., Almquist, J., Webright, W., \& Freeman, J. (2001). Monitoring resolution of postconcussion symptoms in athletes: Preliminary results of a web-based neuropsychological test protocol. Journal of Athletic Training, 36, 280-287.

Ferrara, M. S., McCrea, M., Peterson, C. L., \& Guskiewicz, K. M. (2001). A survey of practice patterns in concussion assessment and management. Journal of Athletic Training, 36, 145-149.

Frerichs, R. J., \& Tuokko, H. A. (2005). A comparison of methods for measuring cognitive change in older adults. Archives of Clinical Neuropsychology, 20, 321-333. 
Grindel, S. H., Lovell, M. R., \& Collins, M. W. (2001). The assessment of sport-related concussion: The evidence behind neuropsychological testing and management. Clinical Journal of Sports Medicine, 11, 134-143.

Guskiewicz, K. M. (2001). Postural stability assessment following concussion: One piece of the puzzle. Clinical Journal of Sports Medicine, 11, 182-189.

Guskiewicz, K. M., Bruce, S. L., \& Cantu, R. C. (2004). National Athletic Trainers' Association position statement: Management of sport-related concussion. Journal of Athletic Training, 39, 280-297.

Guskiewicz, K. M., McCrea, M., \& Marshall, S. W. (2003a). Cumulative effects associated with recurrent concussion in collegiate football players: The NCAA Concussion Study. Journal of the American Medical Association, 290, 2549-2555.

Guskiewicz, K. M., McCrea, M., Marshall, S. W., Cantu, R. C., Randolph, C., Barr, W., et al. (2003b). Cumulative effects associated with recurrent concussion in collegiate football players: The NCAA Concussion Study. Journal of the American Medical Association, 290, 2549-2555.

Guskiewicz, K. M., Ross, S. E., \& Marshall, S. W. (2001). Postural stability and neuropsychological deficits after concussion in collegiate athletes. Journal of Athletic Training, 36, 263-273.

Heaton, R. K., Temkin, N., \& Dikmen, S. (2001). Detecting change: A comparison of three neuropsychological methods, using normal and clinical samples. Archives of Clinical Neuropsychology, 16, 75-91.

Hinton-Bayre, A. D., Geffen, G. M., Geffen, L. B., McFarland, K. A., \& Friis, P. (1999). Concussion in contact sports: Reliable change indices of impairment and recovery. Journal of Clinical and Experimental Neuropsychology, 21, 70-86.

Iverson, G. L., Gaetz, M., Lovell, M. R., \& Collins, M. W. (2004). Cumulative effects of concussion in amateur athletes. Brain Injury, 18, 433-443.

Jacobson, N. S., \& Truax, P. (1991). Clinical significance: A statistical approach to defining meaningful change in psychotherapy research. Journal of Consulting Clinical Psychology, 59, 12-19.

Kane, R. L., \& Reeves, D. L. (1997). Computerized test batteries. In A. M. Horton Jr., D. Wedding, \& J. Webster (eds.), The Neuropsychology Handbook: Vol. 1. Foundations and Assessment, 2nd ed.. New York: Springer, 423-467.

Lovell, M. R., \& Collins, M. W. (1998). Neuropsychological assessment of the college football player. Journal of Head Trauma Rehabilitation, 13, 9-26.

Macciocchi, S. N., Barth, J. T., Alves, W., Rimel, W. R., \& Jane, J. A. (1996). Neuropsychological functioning and recovery after mild head injury in collegiate athletes. Neurosurgery, 39, 510-514.

McCrea, M., Guskiewicz, K. M., \& Marshall, S. W. (2003). Acute effects and recovery time following concussion in collegiate football players: The NCAA Concussion Study. Journal of the American Medical Association, 290, 2556-2563.

McCrea, M., Kelly, J. P., Randolph, C., Cisler, R., \& Berger, L. (2002). Immediate neurocognitive effects of concussion. Neurosurgery, 50, 1032-1040. 
McCrory, P., Johnston, K., \& Meeuwisse, W. (2005). Summary and agreement statement of the 2nd International Conference on Concussion in Sport, Prague 2004. British Journal of Sports Medicine, 39, 196-204.

Mead, A. D., \& Drasgow, F. (1993). Equivalence of computerized and paper-and-pencil cognitive ability tests: A meta-analysis. Psychological Bulletin, 114, 449-458.

Moser, R. S., \& Schatz, P. (2002). Enduring effects of concussion in youth athletes. Archives of Clinical Neuropsychology, 17, 81-90.

Moser, R. S., Schatz, P., \& Jordan, B. D. (2005). Prolonged effects of concussion in high school athletes. Neurosurgery, 57, 300-306.

Notebaert, A. J., \& Guskiewicz, K. M. (2005). Current trends in athletic training practice for concussion assessment and management. Journal of Athletic Training, 40, 320-325.

Schatz, P., \& Zillmer, E. A. (2003). Computer-based assessment of sports-related concussion. Applied Neuropsychology, 10, 42-47.

Temkin, N. R., Heaton, R. K., Grant, I., \& Dikmen, S. S. (1999). Detecting significant change in neuropsychological test performance: A comparison of four models. Journal of the International Neuropsychological Society, 5, 357-369.

Valovich-McLeod, T. C., Perrin, D. H., Guskiewicz, K. M., Shultz, S. J., Diamond, R., \& Gansneder, B. M. (2004). Serial administration of clinical concussion assessments and learning effects in healthy young athletes. Clinical Journal of Sports Medicine, 14, 287-295.

Webbe, F. M., \& Ochs, S. R. (2003). Recency and frequency of soccer heading interact to decrease neurocognitive performance. Applied Neuropsychology, 10, 31-41.

Wilson, B. A., Watson, P. C., Baddeley, A. D., Emslie, H., \& Evans, J. J. (2000). Improvement or simply practice? The effects of twenty repeated assessments on people with and without brain injury. Journal of the International Neuropsychological Society, 6, 469-479.

Witol, A. D., \& Webbe, F. M. (2003). Soccer heading frequency predicts neuropsychological deficits. Archives of Clinical Neuropsychology, 18, 397-417.

Woods, S. P., Childers, M., Ellis, R. J., Guaman, S., Grant, I., \& Heaton, R. K. (2006). A battery approach for measuring neuropsychological change. Archives of Clinical Neuropsychology, 21, 83-89.

Zillmer, E. A.(ed.). (2003). Sports-Related Concussions [Special issue]. Applied Neuropsychology, 10, 1. 